\title{
Depression Involved in the Chemotherapy Induced Event-based Prospective Memory Impairment in Breast Cancer Survivors
}

\author{
Zhonglian Huang \#, Wen Li", Chen Gan, Yue Lv, Haijun Chen, Zhen Yang and Huaidong Cheng*
}

Department of Oncology, the Second Affiliated Hospital of Anhui Medical University, Hefei 230601, Anhui, China \#Co-first author: Zhonglian Huang and Wen Li contributed equally to this work.

\section{*Corresponding to:}

Huaidong Cheng,

Department of Oncology, The Second Affiliated Hospital of Anhui Medical University, No. 678 Furong Road, Hefei Economic and Technological Development Zone, Hefei 230601, China

Tel: +8655163869542, Fax: +8655163869400

Running Title: Prospective Memory Impairment in Breast Cancer Patients

\begin{abstract}
The aim of this study was to investigate the relationships between depression and occurrence of chemotherapy induced prospective memory impairment in patients with breast cancer (BC).The 63 BC patients before and after chemotherapy were administered with the self-rating depression scale (SDS) and a battery of cognitive neuropsychological tests including event-based and time-based prospective memory (EBPM and TBPM,
\end{abstract} respectively) tasks. The changes in their prospective memory and cognitive neuropsychological characteristics before and after chemotherapy were compared. Compared with the scores before chemotherapy, the EBPM score exhibited a statistically significant difference after chemotherapy $(t=6.069, P<0.01)$, while the TBPM score showed no significant difference $(t=1.087, P>0.05)$. Further, compared with the patients without depression, the patients with depression exhibited a statistically significant difference in the EBPM score $(t=-4.348, P<$ 0.01), while the TBPM scores did not show a statistically significant difference between the two groups $(t=-1.260$, $P>0.05)$. Post-chemotherapy, EBPM and overall cognitive functions in $B C$ patients merged with depression were found to decline, while TBPM did not show a www.ijeab.com significant change, suggesting that the combination of chemotherapy and depression might be related with the occurrence of post-chemotherapy EBPM impairment.

Keywords- Breast cancer, depression, cognitive impairment, prospective memory.

\section{INTRODUCTION}

Chemotherapy-related cognitive impairment (CICI) refers to the impairment of cognitive functions-such as memory, information processing speed, and attention- that occurs in cancer patients during or after chemotherapy. Some studies ${ }^{1}$ have suggested that the impact of CICI in longterm survivors of breast cancer (BC) was even worse than the recurrence and metastasis of BC itself, and that it had become an important factor that might affect patients' quality of life. Recently, several studies have examined CICI in BC. Psycho-Oncology, Journal of Clinical Oncology, and other such journals have published the latest research and systematic reviews in this field ${ }^{2,3}$. It was found that $\mathrm{CICI}$ in $\mathrm{BC}$ survivors exhibited significant heterogeneity. Evidently, The CICI in BC have become a hot topic in the field of psycho-oncology.

In recent years, a large number of studies have found that 
BC patients exhibit cognitive impairment after chemotherapy. For instance, Park et al. found that BC patients exhibited subjective and objective cognitive impairments after chemotherapy ${ }^{4}$. Similarly, Wefel et al. found that one or more cognitive functions tend to decline in $\mathrm{BC}$ patients after chemotherapy ${ }^{5}$. Some studies also suggested that the brain exhibited certain heterogeneity after BC chemotherapy. Jim et al. showed that the changes of language and visuospatial functions in the brain were significant after BC chemotherapy ${ }^{2}$. Chen et al. performed multidimensional neuropsychological scale tests on $42 \mathrm{BC}$ patients before and after chemotherapy, and found that the patients exhibited different levels of impairment in memory, attention, social cognition, and other aspects after chemotherapy $^{6}$. Some recent studies have also found that the brain memory impairment was prominent after BC chemotherapy, and that the extent of impairment was also different among different memory components. Cheng et al. found that after BC chemotherapy, the brain exhibited prospective memory (PM) impairment, which mainly manifested as the impairment of event-based prospective memory $(\mathrm{EBPM})^{7}$. Thus, $\mathrm{CICI}$ in $\mathrm{BC}$ has been found to be influenced by a number of factors. Most recently, Paquet et al. studied 80 cases of early BC survivors and found that fatigue after BC chemotherapy could facilitate PM impairment in patients with early BC, and it was thought that the symptoms of fatigue had certain correlation with memory impairment in patients ${ }^{8}$.

$\mathrm{PM}$ is the memory required for future plans or intentions and the memory component most closely related to human daily lives. McDaniel et al. divided PM into event-based prospective memory (EBPM) and time-based prospective memory (TBPM $)^{9}$. Recent studies suggested that the BC patients with CICI presented PM impairment, which was mainly exhibited as EBPM impairment ${ }^{8}$. However, the specific impacting factors are not clear yet. Studies have found emotion to be an important factor affecting memory ${ }^{10}$, and depression was one of the most common mental disorders in $\mathrm{BC}$ patients ${ }^{11}$. Depression could affect physiological functions, treatment compliance, cognitive functions, and quality of life in BC patients ${ }^{12}$. However, whether CICI and PM impairment were related to patients' depression is not clear yet. Particularly, there is no report about whether depression is related to chemotherapyinduced EBPM.

This study attempted to investigate whether chemotherapy and depression in $\mathrm{BC}$ patients were together related to the occurrence of PM impairment. The study subjects included 63 BC patients after chemotherapy. Cognitive neuropsychological tests to assess PM were used to investigate the impact of chemotherapy along with depression on the changes in PM in BC patients.

\section{MATERIALS AND METHODS}

Clinical data of patients enrolled

We selected 63 patients who underwent adjuvant chemotherapy after BC surgery in the Department of Oncology and Department of Beast Surgery, The Second Affiliated Hospital of Anhui Medical University, from October 2013 to August 2014. Out of them, 59 cases were identified as those of invasive ductal carcinoma and 4 as invasive lobular carcinoma by postoperative pathology. The tumor differentiation grade ranged from I-III, with 18 cases in Grade I, 38 in Grade II, and 7 in Grade III. The postoperative staging ranged from I-III. All subjects had undergone at least 5 years of formal education, were righthanded, had normal or corrected vision, and acceptable binaural hearing. Therefore, they all could cooperate with the tests. This study was conducted in accordance with the declaration of Helsinki. This study was conducted with approval from the Ethics Committee of Anhui Medical University. Written informed consent was obtained from all participants.

\section{Grouping criteria}

The following were the inclusion criteria for BC patients: 
had undergone modified BC radical mastectomy; had a clear histopathological diagnosis; initially treated but did not undergo endocrine therapy or brain radiotherapy after surgery; had a life expectancy of $\geq 3$ months; planned for standard FAC chemotherapy after surgery (fluorouracil $500 \mathrm{mg} / \mathrm{m}^{2}$, intravenous infusion on the first day, adriamycin $50 \mathrm{mg} / \mathrm{m}^{2}$, intravenous infusion on the first day, cyclophosphamide $500 \mathrm{mg} / \mathrm{m}^{2}$, intravenous bolus on the first day, with a 21-day cycle for a total of 6 cycles) and conduct normal activities of daily living; had a Karnofsky functional score (KPS) $\geq 80$ points or ECOG score on physical performance within 0 to 1 point; and did not have any hearing, vision, language, or other dysfunction.

The exclusion criteria were having late cachexia or distant metastasis; had undergone endocrine therapy, brain radiotherapy, or other adjuvant therapies; had significant anxiety, depression, and other mental disorders that could disturb their participation in the study; had other physical or mental illnesses that could lead to cognitive impairment; had a history of alcohol or drug dependency; were administered other drugs that could improve cognitive functions, such as donepezil; had intracranial metastases and other abnormalities according to the brain CT or MRI; had an ECOG score of $>2$ points; and had other severe diseases related to the heart, liver, kidney, brain, and hematopoietic system.

\section{Depression assessment}

The SDS was developed by Zung (1965), as a self-rating scale to measure the severity of depression and its changes during treatment. According to Chinese standards, the degrees of depression were divided into 53-62 points: mild depression, 63-72 points: moderate depression, and >72 points: severe depression.

\section{Cognitive neuropsychological tests}

The mini-mental status examination (MMSE), verbal fluency test (VFT), and digital span (DS) were used to assess cognitive functioning. A battery of tests was administered to all subjects to assess general cognitive and memory functions. MMSE was used to assess the cognitive functions, including time and spatial orientation, short-term memory, calculation, language, and visuospatial skills. The Verbal Fluency Test (VFT), in which the subjects were asked to name as many animals as possible within one minute, was used to evaluate the executive function. The Digit Span (DS) test, in which the subjects were asked to recall a series of numbers after hearing them in a randomized order, was used to measure working memory and attention. The total score was determined by the number of digits recalled in a correct serial order.

\section{PM tests}

The EBPM test: Before the test, the subjects were instructed to pick out two words belonging to a subclass from each card in the subsequent trials. When the two selected words represented animals (target word), the subjects had to tap the table once. At the end of the test, the subjects were instructed to speak out their telephone number (without any other hint at the end of the test). The test stimuli included 32 cards with 12 high-frequency and real-meaning Chinese words, among which 10 words belonged to a larger category, while the other 2 words belonged to a subclass. In the learning stage, the subjects were asked to speak out two words of a subclass as the first two cards were for trial. In the trial, the first card did not include the target word, while the second card contained the target word. Following the instructions that were provided before test, when the target word, i.e., a kind of animal, appeared, the subjects' EBPM task was to tap the table once. The other EBPM task was to remember to speak out their phone number when the card selection ended.

Recording method: We referred to the method developed by McDaniel and Einstein ${ }^{13}$. Thus, when a predetermined target word appeared, the subject was expected to perform 
a target behavior, and the number of times the same was correctly executed was recorded as EBPM score. This test comprised a total of six target cards (numbered as 5, 10, 15, 20, 24, and 29). When the target card appeared, the correct response was recorded as 1 point, and remembering to speak out the phone number at end of the test was recorded as 2 points. Thus, the total score on the EBPM test was 8 points.

The TBPM test: Before the test, the subjects were instructed to tap the table once at 5, 10, and $15 \mathrm{~min}$, respectively after the test began, to choose the maximum and minimum numbers from each card. One clock was placed in front of the subjects, such that the subjects could be sure about the time through this clock. The clock was set at 0:00:00 at the beginning of the test. Test stimuli included 100 cards, when the clock started, the subjects were presented the cards one at a time. Each card had 12 double digits, out of which the subjects had to select the minimum and maximum numbers to complete the TBPM. Meanwhile, the subjects were also expected to complete another TBPM task (i.e., taping the table at 5, 10, and 15 min after the commencement of the test). The test was stopped at $17 \mathrm{~min}$.

Recording method: Referring to the method developed by McDaniel and Einstein ${ }^{13}$, the number of times the target behavior was correctly executed at correct time intervals was recorded as the TBPM score. If the action was performed within 10 seconds before or after each target time, it would be record as 2 points. Further, if it was performed within 30 seconds, it was recorded as 1 point. Thus, the total score on the TBPM test was 6 points.

\section{Statistical analysis}

The SPSS 17.0 software was used for data processing. Test scores before and after chemotherapy were compared using a paired-sample t-test, while the general information and neuropsychological characteristics between the depression and non-depression groups were compared using two independent samples t-tests.

\section{RESULTS}

\section{General information}

In total, $63 \mathrm{BC}$ patients were evaluated after chemotherapy, out of which, 29 patients were found to have depression, while 34 patients were not. There was no significant difference in age $(50.66 \pm 7.76$ vs. $47.35 \pm 8.56)$ and years of education $(7.90 \pm 3.36$ vs. $9.44 \pm 3.57)$, and other general characteristics between the depression and nondepression groups $(P>0.05)$.

Comparison of depression, neuropsychological tests, and $P M$

Compared with the results before chemotherapy, SDS and MMSE scores exhibited a statistically significant difference after chemotherapy, while frontal fluency and DS showed no statistically significant differences.

\section{Table 1}

Further, as compared with the results before chemotherapy, the EBPM score exhibited a statistically significant difference after chemotherapy $(\mathrm{t}=6.069, P<0.01)$, while the TBPM score showed no significant difference $(\mathrm{t}=$ $1.087, P>0.05)$

\section{Table 2}

\section{Comparison of neuropsychological tests and PM}

After chemotherapy, the MMSE and VFT scores of the depression group were found to be significantly lower than those of the non-depression group, while the DS score showed no statistically significant difference.

\section{Table 3}

Further, as compared with the depression group, the EBPM score of the non-depression group was significantly different $(\mathrm{t}=-4.348, P<0.01)$, while the TBPM score showed no significant difference $(\mathrm{t}=-1.260, P>0.05)$.

Table 4

\section{DISCUSSION}

Currently, CICI in BC patients is one of the major issues 
concerning the field of psycho-oncology. The high incidence and relatively long survival time of $\mathrm{BC}$ has provided an important window for studies on $\mathrm{CICI}$ in $\mathrm{BC}$ patients. Existing studies suggested that $\mathrm{CICI}$ in $\mathrm{BC}$ patients was mainly related to the following areas: memory, attention, executive function, information processing speed, language function, and visuospatial function ${ }^{14-16}$. The present study found that $\mathrm{BC}$ patients exhibited an overall decline in cognitive functions after chemotherapy, which is similar to the findings of other studies ${ }^{15,16}$. Meanwhile, we also found that chemotherapy, when combined with depression, might be associated with EBPM impairment.

Memory is one of the important human cognitive functions, which refers to the psychological processes through which the brain accumulates and preserves individual experiences. Therefore, it has a prominent place in all human mental activities. According to the time of occurrence of an event, memory could be divided into retrospective memory (RM) and $\mathrm{PM}^{13}$. Other than $\mathrm{RM}, \mathrm{PM}$ is one of the most important forms of human memory. EBPM refers to the memory required to carry out certain actions when a specific event occurs, for example, remembering to go shopping after work. Volle et al. found that the spontaneous extraction of EBPM information was related to the functions of the prefrontal cortex ${ }^{17}$. In one of our previous studies, we found that the patients with frontal lobe impairment tended to exhibit significant EBPM impairment ${ }^{18}$. The present study suggested that the $\mathrm{BC}$ patients with depression had significant EBPM impairment after chemotherapy, which might be related with the changes in the prefrontal structures and insufficient functioning of the same.

Depression is a common mental state in women with BC. Since the 1960s, foreign countries have paid attention to BC women's depression. Fann et al.retrospectively analyzed the occurrence of depression within 1 year after $\mathrm{BC}$ diagnosis over the last 20 years $^{19}$, and found that its incidence after BC surgery was about $10 \%$ to $25 \%$.
Similarly, Luutonen et al. ${ }^{20}$ studied and reported that the incidence of depressive symptoms in patients with newly diagnosed BC was up to $32.1 \%$. Further, in $19.4 \%$ of them the depression was moderate or mild, while in $12.7 \%$ patients it was moderate or severe. Chemotherapy is one of the important treatment to $\mathrm{BC}$ patients. However, most BC patients have had a certain fear towards chemotherapy. Additionally, the incidence of depression in BC patients after chemotherapy has been reported to be even higher than patients before chemotherapy. Lekovich et al. ${ }^{21}$ statistically analyzed the incidence of depression in $95 \mathrm{BC}$ patients within 1-6 months after chemotherapy, and reported it to be up to $67 \%$. Recently, certain studies have found that depression in BC patients was not temporary, and some patients have even exhibited high levels of depressive symptoms after the completion of treatment ${ }^{22-24}$. Studies have also found that not all BC patients suffer from depression, and that those with depression have also exhibited differences in the degree, manifestation, and duration of depression ${ }^{19-21}$. The main causes of depression in $\mathrm{BC}$ patients have been reported as follows: shock from the $\mathrm{BC}$ diagnosis; special importance of breasts to females, and as most $\mathrm{BC}$ patients prefer surgical treatment, the physical change would result in a double whammy physically and mentally; socio-demographic factors such as age, educational level, employment status, household income, obesity, and marital status (all these have been reported to affect patient's depression; however, this idea is still controversial); the side effects of treatment, such as the impact of chemotherapy on fertility, sexual function, perimenopausal period, and related health problems, which have been reported to cause significant anxiety and pain in BC patients ${ }^{25}$. The present study found that depression caused by various factors might be involved in the occurrence of CICI in BC patients. The mechanisms of $\mathrm{CICI}$ in BC patients are not clear yet. However, recently there has been active research in this field. Several studies have found that $\mathrm{CICI}$ is manifested differently in BC 
patients, with many impacting factors and complex pathogeneses. Therefore, several multi-disciplinary studies, such as those on molecular biology and neuroimaging, have been conducted. The present study provided evidence for the incidence of EBPM impairment after BC chemotherapy from the perspective of the effect of emotions on memory, thus providing a clinical basis for further exploring the biological mechanisms of EBPM impairment after BC chemotherapy.

Previous studies have confirmed that patients with depression exhibited significant cognitive impairment. The present study found that the MMSE, VFT, and EBPM scores in depressed patients after BC chemotherapy were lower than those in the non-depressed patients. Therefore, we believe that chemotherapy, together with depression, would further aggravate cognitive impairment in BC patients. Particularly, the changes in EBPM were prominent, suggesting that the combined effect of $\mathrm{BC}$ chemotherapy and post-chemotherapy depression might be one of the important factors affecting EBPM impairment after BC chemotherapy.

In conclusion, this study showed that the $\mathrm{BC}$ patients with post-chemotherapy depression would exhibit a decline in their general cognitive function and EBPM, suggesting that depression might be associated with the occurrence of PM impairment after BC chemotherapy.

\section{CONCLUSION}

This study indicated that EBPM and overall cognitive functions in $\mathrm{BC}$ patients merged with depression were found to decline, while TBPM did not show a significant change, suggesting that the combination of chemotherapy and depression might be related with the occurrence of post-chemotherapy EBPM impairment.

Financial Support: This research was supported by the National Natural Science Foundation of China (No. 81372487).

Conflict of interest: All of the authors declare that they have no conflicts of interest regarding this paper.

Acknowledgements: No.

\section{REFERENCES}

[1] Cheung YT, Chui WK, Chan A. Neuro-cognitive impairment in breast cancer patients: pharmacological considerations. Critical reviews in oncology/hematology. 2012;83(1):99-111.

[2] Jim HS, Phillips KM, Chait S, et al. Meta-analysis of cognitive functioning in breast cancer survivors previously treated with standard-dose chemotherapy. Journal of clinical oncology : official journal of the American Society of Clinical Oncology. 2012;30(29):3578-3587.

[3] Chen X, Li J, Ren J, et al. Selective impairment of attention networks in breast cancer patients receiving chemotherapy treatment. Psycho-oncology. 2014;23(10):1165-1171.

[4] Park JH, Bae SH, Jung YS, Jung YM. [Prevalence and characteristics of chemotherapy-related cognitive impairment in patients with breast cancer]. Journal of Korean Academy of Nursing. 2015;45(1):118-128.

[5] Hermelink K. Acute and late onset cognitive dysfunction associated with chemotherapy in women with breast cancer. Cancer. 2011;117(5):1103; author reply 1103-1104.

[6] Chen X, Li J, Zhu C, Li D, Zhang J, Wang K. [Cognitive function in breast cancer patients on chemotherapy: a longitudinal study]. Zhonghua yi xue za zhi. 2014;94(1):27-30.

[7] Cheng H, Yang Z, Dong B, et al. Chemotherapy-induced prospective memory impairment in patients with breast cancer. Psycho-oncology. 2013;22(10):2391-2395.

[8] Paquet L, Collins B, Song X, Chinneck A, Bedard M, Verma S. A pilot study of prospective memory functioning in early breast cancer survivors. Breast. 2013;22(4):455-461. 
[9] McDaniel MA, Lamontagne P, Beck SM, Scullin MK, Braver TS. Dissociable neural routes to successful prospective memory. Psychological science. 2013;24(9):1791-1800.

[10] Lindquist KA, Gendron M, Barrett LF, Dickerson BC. Emotion perception, but not affect perception, is impaired with semantic memory loss. Emotion. 2014;14(2):375-387.

[11] Suppli NP, Johansen C, Christensen J, Kessing LV, Kroman N, Dalton SO. Increased risk for depression after breast cancer: a nationwide population-based cohort study of associated factors in Denmark, 1998-2011. Journal of clinical oncology : official journal of the American Society of Clinical Oncology. 2014;32(34):3831-3839.

[12] Kalender ME, Buyukhatipoglu H, Balakan O, et al. Depression, anxiety and quality of life through the use of complementary and alternative medicine among breast cancer patients in Turkey. Journal of cancer research and therapeutics. 2014;10(4):962-966.

[13] McDaniel MA, Einstein GO. The neuropsychology of prospective memory in normal aging: a componential approach. Neuropsychologia. 2011;49(8):2147-2155.

[14] Duijts SF, Faber MM, Oldenburg HS, van Beurden M, Aaronson NK. Effectiveness of behavioral techniques and physical exercise on psychosocial functioning and health-related quality of life in breast cancer patients and survivors--a meta-analysis. Psycho-oncology. 2011;20(2):115-126.

[15] Phillips KA, Ribi K, Fisher R. Do aromatase inhibitors have adverse effects on cognitive function? Breast cancer research : BCR. 2011;13(1):203.

[16] Shaffer VA, Merkle EC, Fagerlin A, Griggs JJ, Langa KM, Iwashyna TJ. Chemotherapy was not associated with cognitive decline in older adults with breast and colorectal cancer: findings from a prospective cohort study. Medical care. 2012;50(10):849-855.

[17] Volle E, Gonen-Yaacovi G, Costello Ade L, Gilbert SJ, Burgess PW. The role of rostral prefrontal cortex in prospective memory: a voxel-based lesion study. Neuropsychologia. 2011;49(8):2185-2198.

[18] Cheng HD, Wang K, Xi CH, Niu CS, Fu XM. Prefrontal cortex involvement in the event-based prospective memory: evidence from patients with lesions in the prefrontal cortex. Brain injury. 2008;22(9):697-704.

[19] Fann JR, Thomas-Rich AM, Katon WJ, et al. Major depression after breast cancer: a review of epidemiology and treatment. General hospital psychiatry. 2008;30(2):112-126.

[20] Luutonen S, Vahlberg T, Eloranta S, Hyvari H, Salminen E. Breast cancer patients receiving postoperative radiotherapy: distress, depressive symptoms and unmet needs of psychosocial support. Radiotherapy and oncology : journal of the European Society for Therapeutic Radiology and Oncology. 2011;100(2):299-303.

[21] Levkovich I, Cohen M, Pollack S, Drumea K, Fried G. Cancer-related fatigue and depression in breast cancer patients postchemotherapy: Different associations with optimism and stress appraisals. Palliative \& supportive care. 2015;13(5):1141-1151.

[22] Seliktar N, Polek C, Brooks A, Hardie T. Cognition in breast cancer survivors: hormones versus depression. Psychooncology. 2015;24(4):402-407.

[23] Kesler SR, Blayney DW. Neurotoxic Effects of Anthracycline- vs Nonanthracycline-Based Chemotherapy on Cognition in Breast Cancer Survivors. JAMA oncology. 2016;2(2):185-192.

[24] Pullens MJ, De Vries J, Van Warmerdam LJ, Van De Wal MA, Roukema JA. Chemotherapy and cognitive complaints in women with breast cancer. Psycho-oncology. 2013;22(8):1783-1789. 
[25] Andrykowski MA, Burris JL, Walsh E, Small BJ, Jacobsen PB. Attitudes toward information about genetic risk for cognitive impairment after cancer chemotherapy: breast cancer survivors compared with healthy controls. Journal of clinical oncology : official journal of the American Society of Clinical Oncology. 2010;28(21):3442-3447.

[26] Department of Oncology, the Second Affiliated Hospital of Anhui Medical University, Hefei 230601, Anhui, China

Table.1: Comparison of depression and neuropsychological background testing between the 2 groups before and after

\begin{tabular}{llccll}
\multicolumn{5}{c}{ chemotherapy } \\
\hline Group & $\mathrm{N}$ & SDS & MMSE & VFT & DS \\
\hline Before chemotherapy & 63 & $37.44 \pm 6.74 \#$ & $27.84 \pm 1.73 \#$ & $11.32 \pm 1.59$ & $7.66 \pm 0.60$ \\
& & & & & \\
After chemotherapy & 63 & $48.31 \pm 13.09$ & $26.19 \pm 1.77$ & $11.27 \pm 1.71$ & $7.67 \pm 0.51$
\end{tabular}

Note: \# P<0.05. Abbreviations: SDS, the self-rating depression scale; MMSE, mini-mental state examination; VFT, verbal fluency test; DS, Digit span.

Table.2: Comparison of EBPM and TBPM scores between the 2 groups before and after chemotherapy

\begin{tabular}{llll}
\hline Group & $\mathrm{N}$ & $\mathrm{EBPM}$ & TBPM \\
\hline Before chemotherapy & 63 & $3.10 \pm 1.12 \#$ & $4.95 \pm 0.99$ \\
After chemotherapy & 63 & $1.87 \pm 1.14$ & $4.75 \pm 1.14$ \\
\hline
\end{tabular}

Note: \# $P<0.01$. Abbreviations: EBPM, event-based prospective memory; TBPM, time-based prospective memory.

Table.3: Comparison of neuropsychological background testing between the depression group and the non-depression group after chemotherapy:

\begin{tabular}{lllll}
\hline Group & $\mathrm{N}$ & MMSE & VFT & DS \\
\hline Depression group & 29 & $25.14 \pm 1.19 \#$ & $10.69 \pm 1.50 \#$ & $7.55 \pm 0.57$ \\
Non-depression group & 34 & $27.01 \pm 1.67$ & $11.76 \pm 1.76$ & $7.76 \pm 0.43$
\end{tabular}

Note: \# P<0.05. Abbreviations: MMSE, mini-mental state examination; VFT, verbal fluency test; DS, Digit span.

Table.4: Comparison of EBPM and TBPM scores between the 2 groups after chemotherapy

\begin{tabular}{llll}
\hline Group & $\mathrm{N}$ & $\mathrm{EBPM}$ & TBPM \\
\hline Depression group & 29 & $1.28 \pm 0.96 \#$ & $4.55 \pm 1.18$ \\
Non-depression group & 34 & $2.38 \pm 1.04$ & $4.91 \pm 1.08$ \\
\hline
\end{tabular}

Note: \# $P<0.01$. Abbreviations: EBPM, event-based prospective memory; TBPM, time-based prospective memory. 